\title{
Salud y ambiente en el proceso de desarollo
}

\author{
Health and environment in the process \\ of development
}

Guy Duval 1

\begin{abstract}
We propose a constructivist systemic approach to study relations concerning Health, Environment and Development. Traditional disciplinary approaches are shown to be insufficient in providing comprehensive explanations for specific problems in this concern. From an integrative conceptualization of these issues, we justify our particular theoretic-methodological kind of analysis which we briefly present. Key words Complex Systems; Interdisciplinarity; Environmental and Health Development
\end{abstract}

Resumen Se plantea un enfoque constructivista de análisis sistémico para el estudio de las relaciones entre Salud, Medio Ambiente y Desarrollo. Los diseños disciplinarios habituales se revelan insuficientes para su explicación y comprensión. Partiendo de una conceptualización integradora de ciertos problemas de la Salud, el Medio Ambiente y el Desarrollo, se justifica el recurso a este enfoque teórico-metodológico, que es presentado de manera resumida.

Palabras clave Sistemas Complejos; Interdisciplinariedad; Desarrollo Ambiental y de Salud

1 Centro de Pesquisa Interdisciplinar em Humanidade do México. Primeira Calle de Alumiño 17, Colonia, Maza, México, DF, C.P. 06270, México. dolores@servidor.unam.mx 


\section{Introdución}

El análisis de las relaciones entre Salud, Ambiente y Desarrollo puede efectuarse desde distintos puntos de vista. Si se prioriza el desarrollo, la salud colectiva y el medio ambiente adquieren un valor instrumental, se transforman en medios para alcanzar el primero. La salud colectiva entonces, se administra de manera que las políticas que la regulan, sean óptimas para garantizar la sustentabilidad económica del desarrollo. El medio ambiente se explota, sin que se tomen en cuenta las repercusiones ambientales de los procesos productivos, con los cuales se persigue un desarrollo concebido como crecimiento macro-económico. Las relaciones entre Salud, Ambiente y Desarrollo cambian, si al contrario, el esquema de desarrollo persigue la elevación de la calidad de vida de los grupos humanos involucrados en los procesos productivos, aplicados a un medio ambiente físico, cuya conservación y renovación, son necesarias para la sustentabilidad y equidad de los procesos socioeconómicos y ambientales.

Se contraponen estos dos tipos de proyecto de desarrollo. Uno administra la salud para el desarrollo y el otro plantea, el desarrollo para la salud y el bienestar colectivos. Uno persigue el desarrollo con ganancias máximas, sin importar las repercusiones socio-ambientales y el otro busca un desarrollo sustentable en términos físicos y sociales.

Además de los foros políticos y económicos, estos temas son discutidos en diversos ámbitos científicos, tanto de las ciencias sociales como de algunas ciencias de la naturaleza. Sin embargo, no hay acuerdo en relación a la manera de estudiar los problemas que subyacen estos debates. Esta última afirmación, no implica que los rezagos y deterioros de la salud y del bienestar social, así como un balance negativo entre la explotación y la conservación del ambiente físico, deriven sólo de errores científicos y/o tecnológicos. Los sistemas y regímenes socioeconómicos y políticos de nuestras sociedades desiguales, son los que encierran los principales mecanismos de tales situaciones. Tampoco se pretende minimizar, ni invalidar excelentes análisis y programas realizados, con distintos enfoques teórico-metodológicos, dentro de variadas disciplinas que se han ocupado de tales problemas.

No es defendible que haya una sola manera de estudiarlos. Al contrario, es deseable que las búsquedas teóricas, se multipliquen y enriquezcan los conocimientos y esquemas explicativos de situaciones y procesos, que afectan gravemente a grandes masas poblacionales $y$ el medio ambiente de una manera local y global, a veces por medio de mecanismos apenas sospechados e insuficientemente aprehendidos. El enfoque teórico-metodológico, tema de este trabajo, se aplica a un recorte de la realidad, integrado con situaciones y procesos heterogéneos, que involucran la Salud, el Medio Ambiente y el Desarrollo, cuyo estudio requiere, la aprehensión de mecanismos complejos y dinámicos para explicar su funcionamiento. Este enfoque ha sido evaluado en estudios empíricos, a manera de verificación de la aplicabilidad de desarrollos teóricos a distintos problemas (Garcia, 1988; Tudela et al., 1992). Ofrece a la vez, una ruta de investigación interdisciplinaria, alejada de los diseños habituales y un planteamiento teórico, para el estudio de sistemas complejos con una perspectiva constructivista.

\section{Conceptualización de salud, medio ambiente y desarrollo}

Cierto enfoque epistemológico, considera que el motor principal del desarrollo científico, es el descubrimiento de nuevos problemas y las transformaciones del marco epistémico y no los hechos experimentales bien verificados, que se acumulan y sustituyen otros conocimientos previos, imprecisos e inexactos. Dicho de otro modo, los nuevos conceptos e hipótesis guían la búsqueda empírica.

En relación a la conceptualización de la salud, entendida ésta en su dimensión colectiva y considerando los métodos utilizados para su estudio, la diversidad de puntos de vista, actuales y pasados, refleja una activa evolución. Ya casi nadie piensa que las enfermedades son un castigo divino. Pocos se atreverían a definir la salud como ausencia de enfermedad. Dentro de esta visión médica, hay dificultad en establecer la frontera entre salud y enfermedad, a escala individual y social. Tal dificultad, no surge solamente por la limitación del conocimiento, sino también, como la expresión de la evolución histórica de los conceptos de salud y de enfermedad. Por ello, en la actualidad, se habla preferentemente del proceso salud-enfermedad y del bienestar bio-psico-social del individuo o del grupo social, to- 
mado éste como una condición positiva, dinámica (histórica). Sin embargo, constatamos a través de la literatura especializada, que los conceptos más amplios y avanzados de saludenfermedad, no son universalmente compartidos.

En relación a la explicación del proceso salud-enfermedad, una visión puramente biologizante tropieza con serias objeciones y tiene muy poca aceptación en el medio médico y el no-médico. Si a fines del siglo XIX, por extensión de la teoría del germen (compatible con una visión biologizante), se propuso que para cada enfermedad y condición de salud, había una causa específica que debía ser descubierta y controlada, ahora se admite sin dificultad, las insuficiencias de esta tesis de la singularidad causal. "No obstante, es cierto que a pesar de la aceptación general del concepto de multiplicidad de causas, (...) la atención se centra sobre el agente particular, el factor de riesgo o el vehículo; es esto lo que se considera como causa, mientras que se tiende a ignorar el complejo total de factores sociales y ambientales que crean la causa y la ponen en contacto directo con la posible víctima" (Terris, 1980). Este autor, advierte también, que "la mejor tradición epidemiológica" no cae en este error.

Dentro y fuera de esta tradición, los resultados de las investigaciones han constituido avances importantes y han representado grandes beneficios para la salud colectiva e individual humana. Sin embargo, sus resultados pueden ser contradictorios entre sí y motivo de discusiones entre especialistas. ¿Cómo explicar esta situación? ¿Los desacuerdos resultan de divergencias metodológicas? Si un mismo concepto de salud debiera sugerir modelos explicativos similares, ¿Porqué investigadores guiados por una misma teoría, no alcanzan los mismos resultados al abordar un mismo tema? Una explicación puede ser la diversidad metodológica (por método se entiende los criterios de establecimiento del marco epistémico y de las fases ulteriores de la investigación y no solamente un conjunto tipificado de procedimientos de diseño de protocolos, de investigación de campo y de análisis de datos). $\mathrm{Si}$ al contrario, la metodología es similar, las diferencias en los resultados de estudios de un tema, podrían ser explicadas por hipotéticas divergencias en las bases teóricas de las cuales parten los investigadores.

Con el objetivo de empezar a entender algunas razones de estas divergencias, regrese- mos a Milton Terris. Llamamos la atención sobre la parte final de la cita, porque revela ciertas bases epistemológicas que se pueden compartir o no. La causa de una condición de salud-enfermedad, en su multideterminación, parecería tener una existencia propia, objetiva, independiente del sujeto que investiga y explica, que debe ser descubierta; sería de nuevo una especie de etapa final condensada de un tejido causal que actúa directamente sobre una víctima (individuo o grupo social).

Con este ejemplo, se plantea la necesidad de una reflexión epistemológica, acerca de la explicación de la salud colectiva dentro de las disciplinas que la estudian. Numerosos autores, en distintos ámbitos geográficos y medios científicos, se esfuerzan por ampliar las bases conceptuales y buscar nuevas vías metodológicas. Hay todavía insatisfacción ante las prácticas de investigación tomadas aisladamente (Csereznia Costa et al., 1990; Almeida Filho, 1989; Breilh et al., 1990; Susser, 1977; Laurell, 1989). Una revisión parcial de la literatura, muestra que existen diferencias de un autor a otro, incluso cuando exponen un mismo planteamiento teórico-metodológico (caso de la teoría de sistema complejo en los estudios de la salud colectiva) (Almeida Filho, 1989; Breilh et al., 1990).

El concepto de deterioro ambiental, abarca la contaminación del medio físico y/o la pérdida de recursos para la producción. En general, se piensa primero en los procesos de contaminación. Deterioro y contaminación, casi se hacen sinónimos. El agotamiento de los recursos naturales, utilizados para la producción de satisfactores no es tomado en cuenta por muchos.

La contaminación ambiental, es un asunto importante a nivel local y global. Su estudio es guiado por marcos epistémicos variados y se aborda con diversos métodos dentro de los límites de distintas disciplinas. Alcanza una dimensión planetaria y una magnitud tal, que, más además de afectar la salud de ciertos grupos humanos, se habla actualmente de la sobrevivencia de nuestra especie y de muchas otras amenazadas por los esquemas de explotación de los recursos naturales adoptados. El enfoque ecológico moderno, encierra una gran riqueza teórica para entender y rectificar hasta determinado punto, ciertos efectos de algunas prácticas tecnológicas y científicas. Pero, a veces se limita a condenar la ciencia y la tecnología, como si la conservación del medio fí- 
sico tuviera que ser el único objetivo de las acciones humanas, como si la naturaleza debiera ser servida por el hombre y no al revés.

Superar el problema de la contaminación y buscar un balance positivo durable entre la explotación de los recursos y su conservación, obligan a pensar en las consecuencias lejanas y posibles de acciones humanas para el desarrollo. Esto introduce la idea de un mundo cambiante, que puede ser alterado más allá de ciertos límites desfavorables para las sociedades humanas, versus la idea de un mundo definitivo, dado para siempre, especie de marco intangible de la historia social. Para conocer este mundo, sobre todo para actuar sobre él, es necesario adoptar un enfoque integrador, sin caer en un holismo inútil y falso.

Investigar y actuar sobre el Medio Ambiente para la Salud y el Desarrollo, obliga a jerarquizar algunos problemas relativos a la contaminación y a la conservación de los recursos naturales para un desarrollo sustentable; el enriquecimiento de las bases naturales de la actividad humana y una constante mejoría de la calidad de vida. Dichos problemas varían de un continente a otro, según las regiones geo-económicas, de un país a otro, en función de las tecnologías, de las ideologías, de los sistemas sociales, legales y regímenes políticos vigentes. En cada estudio particular, para establecer una solución transitoria a un problema particular, es necesario un acercamiento específico, distante de todo discurso que quiera dictar normas universales y principios generales, que al final tienen poca utilidad.

Diagnosticar y comprender el deterioro ambiental, en algunos países alude a la deforestación, la ganaderización extensiva, a ciertos modelos de agricultura comercial - monocultivo, mecanización del campo, uso de insumos químicos en el campo - que deben alcanzar un grado de competitividad a escala mundial o regional, partiendo de recursos naturales a veces limitados. La modernización productiva (ej.: pasar de la producción para el autoconsumo, a la producción comercial) en determinadas condiciones socio-ambientales, puede conllevar a la ruptura de modelos socioeconómicos y culturales: cambios de la tenencia agraria, de los mecanismos de financiamiento de la producción, movimientos migratorios masivos - tan conflictivos hoy en día-, entre otros. Esta somera lista se refiere sólo a algunos aspectos de la producción primaria en el medio rural.
En la esfera de las actividades económicas secundaria y terciaria, los efectos urbanos locales, regionales y globales, tanto sobre el medio físico como el medio social, a veces son considerables: contaminación de los suelos, de los cuerpos y cursos del agua, de la atmósfera local y planetaria, crecimiento desmesurado de las urbes, entre otros.

Sin adoptar una visión catastrofista, debe aceptarse que el problema es complejo. No se vislumbran soluciones mágicas. Su comprensión cabal requiere de esfuerzos sostenidos tanto en el terreno de la acción práctica como de la investigación científica, y no solamente tecnológica. Para actuar sobre los problemas actuales y prevenir otros, los mismos principios teórico-metodológicos deben ser revisados.

Es necesario también reconceptualizar el Desarrollo. Éste ya no puede ser visto como un simple crecimiento económico continuo, ni como una acumulación ininterrumpida de saberes científicos y tecnológicos. Sus principios orientadores, son la calidad de vida como objetivo primordial, al mismo tiempo que se admite y se rescata la especificidad de cada sociedad humana y de cada región. Debe ser entendido, como un proceso dinámico con rupturas estructurales y reestructuraciones sucesivas, capaz de afectar intensamente de manera favorable o desfavorable el medio ambiente físico y social.

Además, este concepto encierra una dimensión ética e histórica en todos sus aspectos. Esta búsqueda, difícilmente podrá evitar la polémica social, política e ideológica, debido a la necesidad de lograr un acceso equitativo a los bienes materiales y culturales en la democracia, y el carácter político de tales procesos. Sin embargo, las sociedades humanas contemporáneas, están en principio predispuestas a aceptar que es legítimo reclamar un Desarrollo Integral, como expresión de los logros de una sociedad justa para todos sus miembros. No existe un modelo de desarrollo universal, aplicable a todas las sociedades. Por eso, las búsquedas y los logros de un justo equilibrio entre la Salud, el Medio Ambiente y el Desarrollo, no pueden ser el apanage de un solo sector social. Serán el fruto de una participación social amplia y democrática, en el mejor sentido de la palabra. 


\section{Necesidad de una propuesta metodológica para el estudio de las relaciones entre salud, medio ambiente y desarrollo}

En la actividad científica, se tiene mayores éxitos cuando además de perseguir resultados inmediatos, se reflexiona sobre los fundamentos teóricos del propio quehacer científico y de la ciencia en general. Es posible que algún super especialista no sienta esta necesidad. Pero, sabemos que tarde o temprano se cuestionarán los fundamentos teórico-metodológicos de su quehacer. Algunos científicos procuran escapar de la limitación de la técnica a ultranza. La historia de las ciencias, es la de aquéllos que inventaron mejor fuera de los senderos conocidos, al opuesto del sentido común y con una clara conciencia de las limitaciones de sus teorías e instrumentos.

El investigador científico, debe transformarse un poco en epistemólogo. A medida que uno se aleja de las ciencias naturales, y se adentra en las ciencias sociales, donde las intervenciones humanas confieren un cierto carácter teleológico a las empresas cognoscitivas, se comprende que, sin la reflexión epistemológica, las ciencias sociales se frenan en su desarrollo. La adopción cada vez más extensa del análisis de sistema complejo, se inscribe en esta línea de reflexión. ¿Se trata simplemente de una moda, o corresponde a una necesidad de teorizar?

Cuando se quiere hablar de sistema, no es fácil ponerse de acuerdo (aunque sea parcialmente) sobre este concepto. Esta dificultad aumenta, cuando se trata de sistemas complejos. Este concepto, rompe paradigmas en numerosas ciencias y sin embargo, cada día más investigadores plantean la complejidad de sus objetos de estudio. Al mismo tiempo, la diversidad de visiones hace que cualquier debate sobre los sistemas complejos se vuelva un asunto complicado. Este concepto, encierra profundas diferencias de un campo de conocimiento a otro, y dentro de un mismo campo; de una corriente de investigación a otra. Esta diversidad, indica la tarea de buscar puntos de acuerdo y de enriquecimiento mutuo. La distancia entre las teorías de sistemas, a veces es tal, que uno se pregunta cómo es posible seguir designándolas con el mismo vocablo.

Sin debatir sobre estas diferencias, sólo basta indicar que un gran número de publicaciones y de coloquios, han sido organizados con el objetivo de caracterizar estas corrientes. Por otro lado, han proliferado las instituciones con financiamientos elevados, dedicadas al estudio de los sistemas complejos así como de la complejidad en el nivel teórico y aplicativo en las diversas disciplinas. Sin embargo, debe alertarse a aquéllos que se interesan por mayores informaciones, que al encontrarse con una terminología similar (idéntica a veces) bajo la pluma de diversos investigadores y autores, aquélla se utiliza con contenidos distintos, cubriendo conceptos diferentes.

De manera general, el enfoque sistémico considera los niveles de organización del objeto de estudio; el comportamiento o funcionamiento del mismo, como una entidad integrada, distinto de los comportamientos de sus componentes. Dentro de ciertas corrientes, la probabilidad y la aleatoriedad se han perfilado como respuestas metodológicas de investigación.

Lejos de los preceptos cartesianos de evidencia, reducción, determinación y exhaustividad, actualmente son numerosos los nuevos caminos de la ciencia y entre ellos, el enfoque sistémico se ha fraguado un espacio todavía en fase de expansión. Sin embargo, tal enfoque metodológico no es aplicable en cualquier investigación. Las disciplinas que estudian aspectos precisos y estrechos de la realidad, no tendrían porqué recurrir a él. Por ejemplo, algunos experimentalistas de laboratorio comparten esta situación, aunque hablen a veces de sistema al referirse a su objeto de estudio (los elementos de la complejidad en inmunología son el gran número, la diversidad y las combinatorias posibles). El concepto de complejidad varía según se sitúe el investigador en la Física, en la Biología, en las Ciencias Sociales (Sociología, Administración, Economía) o en las Ciencias Cognitivas. Ciertas corrientes conciben lo complejo como algo natural dado y otros como algo construido. Luego, complicación y complejidad se hacen sinónimos.

$\mathrm{Al}$ aceptar que el enfoque de sistemas complejos, constituye una alternativa teórico-metodológica para la investigación de numerosos problemas, debe evitarse caer en la posición de aquéllos que, paradójicamente, por la misma razón invocada por los adeptos del enfoque sistémico, buscan una integración imposible de la realidad, queriendo relacionar todo con todo.

Para presentar una perspectiva constructivista de los sistemas complejos, es útil, en un 
principio, prestar atención a lo planteado por los investigadores que rechazan el enfoque de sistema, por contradecir éste sus propios planteamientos teóricos.

Consideremos cierta forma de reduccionismo, que asume que para conocer el objeto de estudio, basta el análisis de sus partes más simples. Tal planteamiento, constituye la antítesis de cualquier visión sistémica. Si se postula que algo es conocible sólo a través de las características de sus componentes, este algo (aunque establecido como una entidad perfectamente identificable) no tendrá más propiedades que las que puedan deducirse de éstos.

Sin embargo, otros científicos, que se reclaman como reduccionistas, podrían insistir en que tienen éxito al disecar la realidad en partes interactuantes, para luego organizarla sistémicamente. Efectivamente, ¿qué se podría objetar a la visión de un diseñador de circuitos eléctricos que conoce por separado cada componente de su circuito, y adopta cierta disposición de los mismos para fabricar aparatos distintos? Se debe admitir que, en este caso, él trabaja con partes que conoce separadamente fuera del dispositivo producido y que es a partir de este conocimiento y estableciendo ciertas relaciones entre dichas partes, que genera distintos "sistemas eléctricos", con características previstas de gran precisión. Este ejemplo sirve para mostrar cómo, al generar entidades diferentes a partir de los mismos elementos, ciertos reduccionistas tendrían razón en insistir que, aunque algo resulte ser mayor que la suma de sus partes, debe ser explicable en función de sus partes y de la organización particular de éstas. Esta postura cuestionaría el concepto de propiedades características de una entidad, inexplicables sobre la base del análisis por separado, de cada una de sus partes constitutivas.

Aquí hay que discernir una diferencia importante entre el conocimiento de algo planeado y realizado por el humano con precisión, con una finalidad determinada y la interpretación, de la realidad en la cual puede estar involucrado. Pero, siempre sin haberla planeado de un modo experimental. Si el estudio de ambos tipos de objetos o procesos, permite inferir relaciones específicas entre sus partes constitutivas, en un caso aquéllas han sido decididas a priori y en el segundo, son elementos de una construcción hipotética de la realidad en el nivel cognoscitivo. En este último caso, las relaciones son supuestos teóricos para explicar la realidad y deben ser verificados a posteriori. Si no se advierte esta diferencia, las relaciones y también los elementos del sistema, aparecen como características invariantes del objeto de estudio y con una existencia real independiente del sujeto investigador. Estas son vistas como propiedades ontológicas del objeto construido y de la realidad y no como atributos puestos por el investigador en ambos casos.

La organización de la realidad como sistema complejo que aquí se expone, prioriza las relaciones dentro del objeto de estudio, entre éste y su entorno, y plantea que ellas derivan de una teoría y en ningún momento pueden ser vistas como partes dadas de la realidad. Por ende, son construcciones del sujeto para explicar la realidad. Cierto empirismo que pretende una lectura directa de la realidad por el investigador, niega aquél enfoque sistémico que construye relaciones, considera que el sistema está dado en la realidad y que sus relaciones sólo deben ser "descubiertas". Es evidente que se está olvidando que las partes del objeto, que aparecen hoy como dadas (evidentes), son algo que a otros niveles y en otros tiempos y espacios, al igual que sus relaciones, tuvieron que ser inferidas (construidas) a partir de una teoría.

A diferencia de algunos enfoques sistémicos, para los cuáles el sistema está dado en la realidad, aquí el sistema complejo es una construcción del sujeto. Las propiedades de un sistema son diferentes de las de sus partes, precisamente, porque son construcciones de un investigador empeñado en explicar la realidad y colocado ante la necesidad de descomponer para conocer.

Al plantear estos problemas, se advierte que cualquier reflexión sobre las teorías de sistemas, termina siendo una discusión epistemológica. Al abordar este tema, no se trata de defender alguna receta para la investigación empírica, más eficaz que otras. Pero, al mismo tiempo se puede admitir que, mientras numerosos temas de investigación son resueltos de manera adecuada con otros métodos y en los límites de una sola disciplina, otros son deformados, si el investigador no alcanza una visión integrada de los diversos aspectos.

Esta última afirmación, ilustra la manera en que ciertos procesos socio-ambientales son tratados a veces. A pesar de admitir, que integran aspectos heterogéneos del medio físico, del medio social, de la esfera productiva y tec- 
nológica, su análisis se limita a menudo a un solo ámbito disciplinario. El especialista no se preocupa por establecer los mecanismos complejos, derivados de interacciones múltiples. Los ignora por pertenecer a campos de saber ajenos a su competencia. Prefiere seguir el camino disciplinario. Por ejemplo, recurrir a esquemas unicausales: las hambrunas, la desnutrición han sido atribuidas por algunos a las sequías, o a la pobreza, o a la explosión demográfica. Una baja producción y reducida productividad agrícolas, expresan la ignorancia del campesino que no sabe seleccionar la tecnología adecuada, entre otros. Al invocar una sola causa, se construyen esquemas explicativos lineales, que no rescatan las interacciones entre los procesos heterogéneos que integran el recorte de la realidad abordada.

Al opuesto de estos planeamientos, se sitúa la obra Drought and Man de Rolando García (Garcia, 1982), relativa a la tristemente célebre hambruna del Sahel de 1972. Constituye una muestra de la riqueza del análisis de sistema complejo desde una perspectiva constructivista.

\section{El sistema complejo}

El concepto de sistema complejo desde una perspectiva constructivista, se presenta aquí con sus características más importantes (Garcia, 1986). Algunas son comunes a distintos enfoques de sistemas, y otras tienen una especificidad bien marcada (Mattessich, 1978; Le Moigne, 1977; Bertalanffy, 1986; Morin, 1986).

1) El sistema complejo, es una propuesta de organización (en el nivel cognoscitivo) de un recorte de la realidad. El investigador selecciona situaciones, fenómenos, procesos e integra con ellos una entidad, que tiene un funcionamiento particular a través de un conjunto de actividades. La selección se hace tomando en cuenta los procesos considerados por él importantes en relación a sus preguntas. Esa aseveración, refuerza la idea de que el sistema no está dado en la realidad y por tanto, no se descubre. Se construye en cada investigación particular y a lo largo de ella. Al inicio de la investigación, el primer esquema de sistema no es más que una hipótesis de trabajo (una respuesta a la pregunta o problema planteado, antes de toda verificación).

2) El sistema complejo se concibe abierto. Guarda relaciones con factores externos que tienen una dinámica propia y autónoma en relación al sistema. El sistema recibe influencias de estos factores y en su interacción con ellos, se dan las condiciones de contorno (condiciones en el límite) del sistema particular construido. Estas condiciones se traducen en flujos de entrada y salida, específicos para el sistema establecido: insumos (créditos, tecnología, materia prima, mano de obra, energía, entre otros), productos (mercancías, emigraciones, entre otros). La interacción del sistema con su entorno lo dinamiza y es la principal generadora de sus cambios. Además, las condiciones de contorno condicionan la estructura que el sistema puede adoptar ante perturbaciones externas al sistema establecido (ej: una nueva tecnología cambia las relaciones de producción). Si la perturbación es asimilable, no hay cambios de estructura, y el sistema conserva un equilibrio dinámico. De otra manera, se desestructura para luego entrar a una nueva fase de estabilización.

La estructura se mantiene en condiciones estacionarias mediante procesos dinámicos de regulación. No tiene un carácter ontológico y las relaciones que la integran no son permanentes. Se establece en correspondencia con las preguntas y problemas planteados, con la visión del investigador, en otros términos, con el marco epistémico de la investigación. Su construcción encierra dificultades, que derivan de la necesidad de articular procesos heterogéneos con escalas temporales y espaciales diversas. Confronta también, el reto de no caer en un idealismo infructuoso y la necesidad de verificar empíricamente las construcciones teóricas. En esta manera de investigar, se descubre que el mejor aliado del investigador de sistemas complejos, es la realidad misma que no es modelizable, interpretable o esquematizable en términos de sistema, de cualquier manera.

El sistema complejo, se concibe como una totalidad organizada en la cuál confluyen procesos heterogéneos. La realidad no está dividida en disciplinas. Así planteado, no es reducible a la simple yuxtaposición de procesos, situaciones o fenómenos del dominio de una disciplina. Se caracteriza por abarcar elementos que pertenecen al dominio material y conceptual de diversas disciplinas. Esto obliga a recurrir a la interdisciplinariedad. Un sistema así integrado, adquiere características propias, diferentes de las de sus partes aisladas. No puede ser representado por una adición de las pro- 
piedades de ellas, aunque resulte de sus interacciones. En este sentido, como totalidad realiza un conjunto propio de actividades, es decir, tiene un funcionamiento propio.

3) El enfoque de sistema complejo, se justifica cuando los problemas abordados traducen la interacción de múltiples factores, es decir, que su visión será incorrecta si se basa en informaciones elaboradas separadamente en campos disciplinarios distintos. No es el número de fenómenos, de procesos o situaciones abarcados, lo que le confiere el carácter de complejo al sistema. Si todo sistema complejo es complicado, no ocurre al revés. Las partes se determinan y condicionan mutuamente, de manera que resultan indisociables una de la otra, o sea que son interdefinibles. Al mismo tiempo, el sistema global resultante es de un nivel jerárquico distinto del de sus elementos constitutivos. Establece relaciones con sus partes, determina y condiciona las características de éstas, que no son aislables una de otra, ni de él. De esta manera se enfatiza la indescomponibilidad del sistema constituido. Si buscáramos un antónimo de sistema complejo, hablaríamos de sistema descomponible y no de sistema simple.

4) La organización alude a la arquitectura de la complejidad. Diversos modelos organizan el objeto de estudio, de modo que sus partes y niveles resulten evidentes (ej: un juego de muñecas rusas, las matrioshkas). Pero, el sistema complejo admite otro tipo de organización, que establece niveles jerárquicos, no evidentes. Incorpora la estratificación de los mecanismos de los cuales se deriva directa o indirectamente el funcionamiento del sistema. Estos, se distribuyen en niveles diferenciados, cada uno con su dinámica propia. Los niveles así concebidos, son semi-autónomos en relación uno a otro y pueden ser estudiados por separado. Hablamos de semi-autonomía porque interactúan entre sí, determinando el nivel superior y las condiciones de contorno del nivel inferior.

5) La visión de la realidad como sistema complejo, obliga a superar la descripción y la explicación sincrónica de una fase estacionaria de cualquier proceso y a verla en su diacronía. Al hablar de estructura del sistema, hemos insistido en que las relaciones del sistema no son propiedades permanentes del mismo. Además de provenir de las características de los elementos del sistema, derivan también, de la historia de éste, que es regulada por la interacción de dichos elementos. Las relaciones sincrónicas de una fase aislada del sistema, no explican porque éste adquirió un determinado estado dinámico. La investigación del sistema complejo, pasa por la diacronía que permite establecer mecanismos evolutivos, para comprender el paso de una fase evolutiva a otra. La reconstrucción evolutiva de un sistema complejo, supera la descripción de estados sucesivos; incorpora los mecanismos complejos que son considerados responsables de sus desestructuraciones y reestructuraciones.

El sistema complejo aparece entonces, como una entidad evolutiva cambiante. Su reconstrucción diacrónica plantea el tema de los principios de su evolución. El estudio de los mecanismos de evolución de cada sistema particular, permite entender cómo se establece y cambia su estructura. Los sistemas complejos cambian por desestructuraciones y reestructuraciones y no de manera gradual y continua. En diversos dominios del saber científico, se ha adquirido la convicción de que el paso de un estado dinámico a otro, se consigue por la vía de la desestructuración o ruptura de la fase anterior. Las desestructuraciones son consecuencias de inestabilidades frente a fluctuaciones debidas a perturbaciones, que superan ciertos umbrales de las propiedades del sistema, como lo son su resistencia al cambio, su flexibilidad. Este planteamiento no se confunde con una posición catastrofista, que exigiría admitir que en cada nueva etapa, uno está en presencia de un sistema nuevo, que no tiene nada que ver con su antecedente. En cada reorganización o reestructuración, el sistema adquiere nuevas relaciones, capaces de fluctuar dentro de ciertos límites, siendo que durante estas etapas de estabilidad relativa, los cambios son previsibles.

En resumen, un sistema complejo, organiza un recorte de la realidad en elementos o subsistemas, que interactúan de modo que ninguno sea definible por separado y en independencia de los otros. De dichas interrelaciones, se deriva la integración del sistema como una entidad, con un funcionamiento propio, influenciado a su vez por las interacciones (condiciones de contorno) con factores externos, cuyos dinamismos son autónomos en relación al sistema. Por estas mismas razones, el estudio de un sistema complejo, debe superar la visión sincrónica y buscar reconstruir su evolución a través de sus cambios estructurales. 


\section{La investigación de sistemas complejos}

Implica el estudio de las interacciones entre fenómenos de dominios disciplinarios distintos. Al no pretender descubrir "hechos dados", ni relaciones "permanentes" de los elementos del sistema, se busca más que la producción de respuestas parciales a problemas parciales. La investigación interdisciplinaria de sistemas complejos, busca la integración del objeto de estudio, que se enriquece con nuevas propiedades y características, en lugar de reducirse a una mera integración de disciplinas. El cometido es rescatar la unidad en la diversidad, la especialidad en la universalidad.

Se puede recurrir a la interdisciplinariedad sin comprometerse con el enfoque teórico-metodológico que hemos presentado. Sin embargo, es parte necesaria del análisis de sistemas complejos. Esto no implica el abandono de los estudios disciplinarios especializados, que son indispensables en la investigación interdisciplinaria de sistemas complejos.

Para guiar la práctica interdisciplinaria, adoptamos el criterio de partir del establecimiento de las interrelaciones, que se dan entre los procesos de la realidad y no de las interrelaciones entre las disciplinas (Garcia, 1994). Los estudios especializados son guiados por un marco epistémico común, establecido previamente a toda búsqueda empírica especializada. En esta exigencia, reside la principal diferencia entre la interdisciplinariedad y la multi o pluridisciplinariedad, que consiste en perseguir a posteriori, alguna correlación entre los hallazgos empíricos de los especialistas, hechos fuera de un marco de referencia común. La multidisciplinariedad, busca las contribuciones de una asociación infinita de especialistas, sólo limitada por los recursos económicos. La transdisciplinariedad, que literalmente busca la coordinación de disciplinas afines, en algún aspecto de sus dominios material y conceptual. En algunas ocasiones, esta coordinación ha resultado en la producción de nuevas disciplinas bien constituidas, como la físico-química, la psico-lingüística, entre otros. A diferencia de estas dos prácticas, en la interdisciplinareidad, hay una gran variación en la asociación de especialistas, que deben seguir haciendo cada uno por su lado lo que saben hacer como especialistas.

La investigación interdisciplinaria, comprende básicamente dos tipos de actividades, que se alternan durante su realización: la in- tegración y de diferenciación. La integración, comienza con el planteamiento del marco epistémico y teórico del problema y consiste, en la propuesta de esquemas sistémicos, que permiten hipotetizar un cierto funcionamiento del objeto de estudio. La diferenciación, se verifica en los estudios disciplinarios empíricos, realizados por cada especialista con sus mejores recursos metodológicos, aplicados a los observables establecidos en función del esquema sistémico, y bajo un mismo marco teórico.

Las interrelaciones entre la salud, el medio ambiente y el desarrollo, constituyen una estructura dinámica y heterogénea, donde los elementos escogidos por el investigador para su integración son interdefinibles. Su conceptualización como un sistema complejo, requiere del abordaje interdisciplinario, que deje atrás las correlaciones lineales entre factores y la simple descripción de estados sucesivos de parámetros aislados. Este planteamiento, representa el inicio de una búsqueda ya emprendida por diversos autores. Encierra la posibilidad de una revisión de la fundamentación de diversas disciplinas que estudian diferentes aspectos de la realidad. 


\section{Referencias}

Almeida Filho N 1989. Epidemiologia sem Números. Uma Introdução Crítica à Ciência Epidemiológica. Associação Brasileira de Pós-Graduação em Saúde Coletiva, Editora Campus Ltda., Rio de Janeiro.

Bertalanffy L von 1986. General System Theory: Essays on its Foundation and Development, Braziller. New York.

Breilh J, Granda E, Campana A, Yépez J, Páez R \& Costales P 1990. Deterioro de la Vida: Un Instrumento para el Análisis de Prioridades Regionales en lo Social y la Salud. Serie Biblioteca de Ciencias Sociales, vol. 28, Centro de Estudios y Asesoría en Salud, Corporación Editora Nacional, Quito.

Csereznia Costa D, Breilh J, Goldberg M, Lima Barreto M, Almeida Filho N, Rosario Costa N \& Mendes Gonçalves RB 1990. Epidemiologia, Teoria e Objeto. Associação Brasileira de Pós-Graduação em Saúde Coletiva, Hucitec-Abrasco, São Paulo.

García R y cols. 1988. Modernización en el Agro: ;Ventajas Comparativas Para Quién? El Caso de los Cultivos Comerciales en el Bajío. International Federation of Institutes for Advanced Studies, Research Series n. 8, Centro de Investigación y Estudios Avanzados (CINVESTAV) - International Federation of Institutes for Advanced Studies (IFIAS) - United Nations Research Institute for Social Development (UNRISD), México.

García R y cols. 1988.Deterioro Ambiental y Pobreza en la Abundancia Productiva: El Caso de la Comarca Lagunera. International Federation of Institutes for Advanced Studies, Research Series n. 9, Centro de Investigación y Estudios Avanzados (CINVESTAV) - International Federation of Institutes for Advanced Studies (IFIAS), México.

García R 1982. Drought and Man, vol. I, Nature pleads not guilty, 1981; García R \& Escudero J, vol. II, The Constant Catastrophe: Malnutrition, Famines and Drought, García R \& Spitz P 1986, vol. III, The Roots of Catastrophe, Pergamon Press, Oxford.
García R 1986. Conceptos básicos para el estudio de sistemas complejos. In E Leff, Los Problemas del Conocimiento y la Perspectiva Ambiental del Desarrollo, Siglo XXI, México.

García R 1994. Interdisciplinariedad y sistemas complejos, In E Leff, Ciencias Sociales y Formación Ambiental, Gedisa, Barcelona.

Laurell AC 1989. Social analysis of collective health in Latin America. Social Science and Medicine 28(11): 1183-1191.

Le Moigne J-L 1977. La Théorie du Système Général. Théorie de la Modélisation, 1ère édition. Presses Universitaires de France, Paris.

Mattessich R 1978. Instrumental Reasoning and Systems Methodology. An Epistemology of the Applied and Social Sciences, D. Reidel Publishing Company, Dordrecht, Holland; Boston, USA; London, England.

Morin E 1986. El Método. I La Naturaleza de la Naturaleza, Cátedra, Madrid.

Susser M 1977. Causal Thinking in the Health Sciences: Concepts and Strategies of Epidemiology, Oxford University Press, New York.

Terris M 1980. La Revolución Epidemiológica y la Medicina Social. Compilación de Ignacio Almada Bay y Daniel López Acuña, traducción de X. Massimi, Siglo XXI, México, 32 pp.

Tudela F 1992. La Modernización Forzada del Trópico: El Caso de Tabasco. Proyecto Integrado del Golfo. International Federation of Institutes for Advanced Studies, Research Series n. 10, Colegio de México, Centro de Investigación y Estudios Avanzados (CINVESTAV) - International Federation of Institutes for Advanced Studies (IFIAS) - United Nations Research Institute for Social Development (UNRISD), 2nda ed., México. 